\title{
The wounded worm
}

\section{Using C. elegans to understand the molecular basis of skin wound healing}

\author{
Suhong Xu, Tiffany I. Hsiao and Andrew D. Chisholm* \\ Division of Biological Sciences; Section of Cell and Developmental Biology; University of California San Diego; La Jolla, CA USA
}

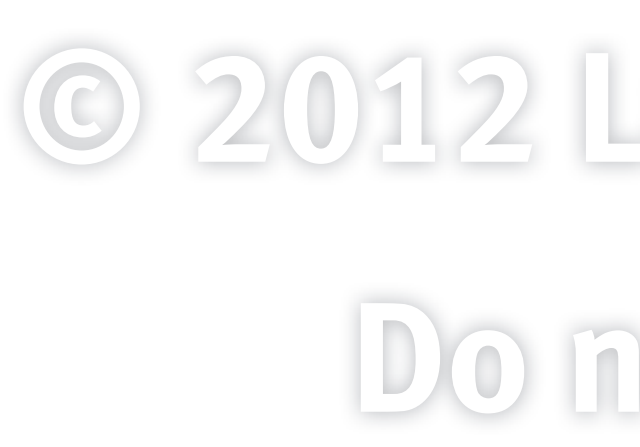

Keywords: epidermis, calcium, innate immune response, AMPs, actin polymerization, filopodia, Rho GTPase, Cdc42, DAPK-1, scar formation

Submitted: 01/06/12

Revised: 01/23/12

Accepted: 01/26/12

http://dx.doi.org/10.4161/worm.19501

${ }^{*}$ Correspondence to: Andrew D. Chisholm; Email: chisholm@ucsd.edu

Commentary to: Xu S, Chisholm AD. A Gaq-Ca ${ }^{2+}$ signaling pathway promotes actin-mediated epidermal wound closure in C. elegans. Curr Biol 2011; 21:1960-7; PMID:22100061; http://dx.doi. org/10.1016/j.cub.2011.10.050
$\mathrm{T}^{\mathrm{k}}$ e ability to heal wounds is an ancient and conserved function of epidermal epithelial layers. The importance of skin wound healing to human life and biology has long been evident, however many of the molecular mechanisms underlying wound repair remain little understood. In the past several years, analysis of the $C$. elegans innate immune response to fungal infection of the epidermis has led to investigations of the ability of the $C$. elegans skin to respond to damage. In a recent paper we used live imaging to investigate the cell biological basis of wound repair in the adult $C$. elegans epidermis. We found that needle or laser injury of the skin triggers a large and sustained increase in epidermal calcium. Epidermal calcium signals appear to specifically promote actin-dependent processes of wound closure. The innate immune and wound closure responses act in parallel to promote survival after injury. Our findings indicate that wounding triggers multiple signals in the $C$. elegans skin. C. elegans offers a tractable model to dissect how epidermal epithelia activate coordinated responses to repair damage.

\section{Introduction}

All multicellular organisms have developed impermeable external skin barriers that form the first line of defense against mechanical damage and pathogenic attack. The ability of skin layers to repair and heal wounds is essential for tissue integrity and animal survival, and may have evolved early in animal evolution. ${ }^{2}$ Mammalian wound healing has been long studied, and has been typically divided into three phases: inflammation and hemostasis, proliferation and migration, and remodeling/maturation. ${ }^{3}$ Although some of the key events in these processes are well described, many underlying molecular mechanisms remain unknown. Understanding these molecular mechanisms is of importance if we wish to develop new strategies to improve wound healing in therapeutic contexts. Several in vivo models have been used to study wound healing, including mouse, zebrafish, Xenopus and Drosophila. ${ }^{2,4}$ However, many aspects of wound repair remain poorly defined at the molecular level. In our recent study we showed that wound closure in the adult $C$. elegans epidermis is dependent on a novel epidermal calcium signal that directs actin polymerization and closure of the wound. ${ }^{1}$

\section{Comparing C. elegans and Mammalian Skin}

The skin layers of mammals and nematodes, while structurally very different, exhibit certain fundamental similarities (Fig. 1). Both are epithelial tissues; differentiated mammalian skin is composed of a multilayered stratified squamous epithelium (epidermis) and an underlying connective tissue layer (dermis), whereas the nematode skin is formed by a simple epithelial layer. The mammalian epidermis is generated from specialized keratinocytes that divide in the basal layer and whose progeny undergo progressive differentiation into the corneocytes of the stratum corneum (SC). The SC forms the primary 
permeability barrier of the skin and is composed of corneocytes embedded in a lipid-rich extracellular matrix., ${ }^{5,6}$ The C. elegans cuticle is in some senses analogous to the SC; it is an extracellular compartment composed of cross-linked collagens, insoluble proteins termed cuticlins, and associated glycoproteins and lipids. ${ }^{7}$ External to the collagenous cuticle are two less well-understood extracellular layers, the lipid-rich epicuticle and the surface coat. The location of the permeability barrier in the nematode skin is not yet clearly defined; it may be formed by a combination of the epidermis, the cuticle and the epicuticle. Among other salient differences, most of the larval or adult C. elegans skin is formed by a single large syncytial cell (termed hyp7); blast cells known as seam cells generate new epidermal cells that fuse with hyp7 allowing postembryonic growth.

\section{The Cutaneous Innate Immune Response to Injury}

Although countless $C$. elegans skin wounds have been caused by DNA or RNA microinjection experiments, it is only recently that the ability of $C$. elegans to survive and repair such drastic trauma has been examined. Evidence that the nematode skin can play an active role in injury responses first came from studies of the fungus Drechmeria coniospora, one of many cuticle-penetrating nematode pathogens whose route of infection is via the skin. Pioneering work from the Ewbank lab revealed that Drechmeria infection induces expression of antimicrobial peptide genes (AMPs) in the epidermis; many of these AMPs had been annotated as neuropeptide-like ( $n l p$ genes), but appear to have a protective role. AMP induction after infection requires the Tollinterleukin receptor like domain protein

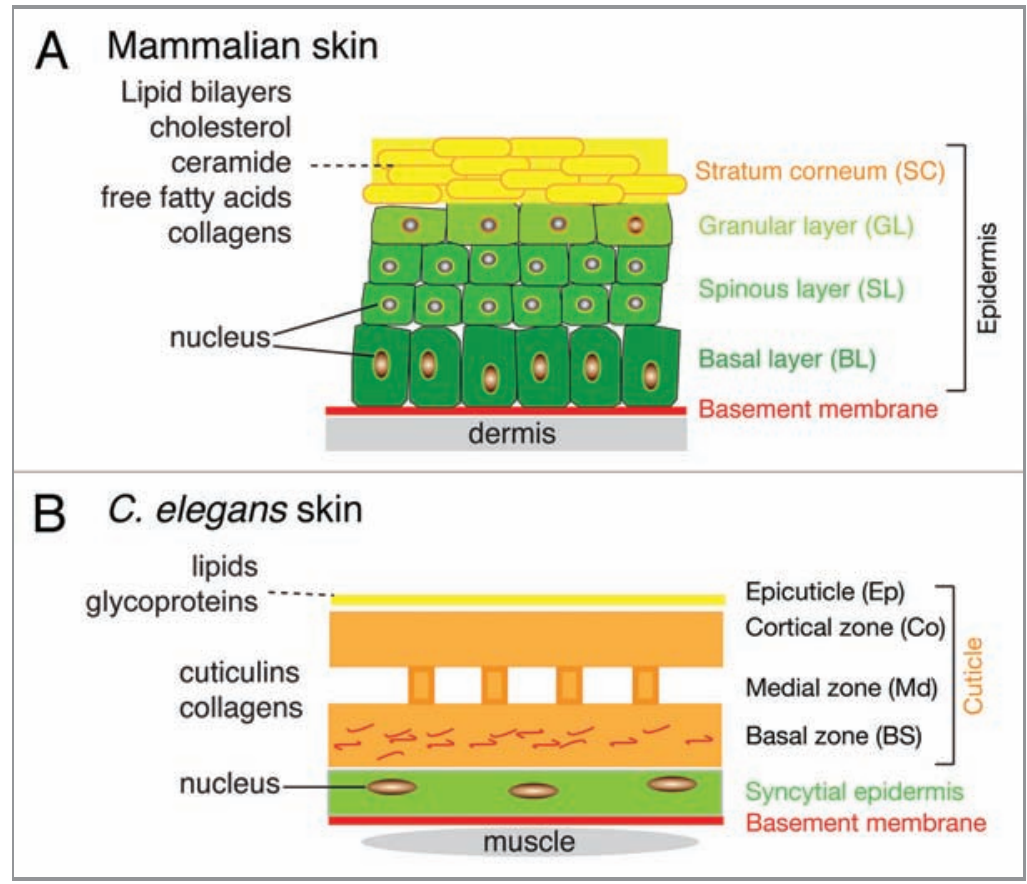

Figure 1. Comparison of mammalian and C. elegans skin layers. (A) Mammalian skin consists of the epidermis and dermis, separated by a basement membrane. The epidermis is composed of several cell layers, including the basal layer (BL) resting on the basement membrane, and the differentiated cell layers of the spinous layer (SL), granular layer (GL) and the stratum corneum (SC). The SC is a lipid-rich layer composed of cholesterol, free fatty acids, ceramides and collagen, which together provide the permeability barrier function of the skin. (B) C. elegans skin consists of the epidermis and cuticle. The epidermis is a simple epithelium whose basal surface rests on a basement membrane. The apical surface of the epidermal epithelium secretes the cuticle, a collagenous extracellular matrix. The cuticle is a flexible barrier layer that is composed predominantly of cross-linked collagens. External to the cuticle is a lipid rich epicuticle that may also function in the permeability barrier.
TIR-1 and the PMK-1 p38 mitogenactivated protein kinase cascade. ${ }^{8,9}$ Further studies of the response to Drechmeria infection identified additional signaling components acting upstream of the TIR-1/p38 cascade, including the G protein GPA-12/G $\alpha 12$, phospholipase C/PLC-3, and TPA-1/PKC $\delta .{ }^{10}$ Infection also activates expression of caenacin AMPs through a neuroendocrine transforming growth factor- $\beta$ (TGF- $\beta$ ) signaling pathway. ${ }^{10}$

The findings that skin infections triggered innate immune responses raised the question of whether such responses were pathogen-specific or a more generic response to damage. These thoughts motivated the development of epidermal wounding procedures involving either small puncture wounds from microinjection needles, or localized damage caused by laser irradiation. ${ }^{9}$ Current needle wounding procedures involve puncturing the cuticle and hyp7 in the posterior body; the epidermal basement membrane and internal organs may also be damaged. Femtosecond laser wounds are more likely confined to the hyp7 cell, although the exact mechanism of damage remains to be fully explored. Thus, current $C$. elegans wounding experiments essentially address the repair capacity of a single large syncytial cell as opposed to wound responses of multicellular epithelia typically studied in other organisms. ${ }^{11}$ Wild type animals display a normal life span after such wounds, and indeed both types of wound can induce AMP expression, often to levels comparable to that seen after infection. Epidermal innate immune responses to injury are also well known in the mammalian skin, which massively upregulates AMPs after injury, independent of infection or inflammation. ${ }^{12}$ In C. elegans, wound-induced AMP expression requires many of the same signaling components as infection-induced expression, although some factors such as the kinase NIPI-3 are only required for the response to infection. ${ }^{9}$ Additional studies of dapk-1 (death associated protein kinase) mutants revealed a syndrome of late-onset epidermal phenotypes comprised of constitutive upregulation of epidermal AMPs and progressive hypertrophy of the cuticle, ${ }^{13}$ suggesting that dapk-1 mutants might 
provide a model for spontaneous epidermal wounding. Taken together, these studies indicated that puncture or laser wounds or the "spontaneous wounds" of dapk-1 mutants can induce epidermal innate immune responses, raising the question of how other aspects of wound repair are regulated.

\section{Wounding Triggers an Epidermal Calcium Signal}

To understand the initiating events in epidermal responses to damage we focused on calcium signaling, long known to be important in embryonic wound healing and in single cell wounding. ${ }^{14,15}$ Using the genetically encoded $\mathrm{Ca}^{2+}$ sensor GCaMP3 expressed in the adult epidermis, we found that wounding triggered a rapid elevation of epidermal $\mathrm{Ca}^{2+}$ that can persist for at least $1 \mathrm{~h}$ (Fig. 2A). When $\mathrm{Ca}^{2+}$ elevation is blocked by soaking worms in the $\mathrm{Ca}^{2+}$ chelator BAPTA-AM, the ability of animals to survive needle wounding is dramatically reduced, suggesting that persistent $\mathrm{Ca}^{2+}$ activation after wounding is required for wound healing. Where does the epidermal $\mathrm{Ca}^{2+}$ come from? Internal stores of $\mathrm{Ca}^{2+}$ in the epidermis may contribute to the $\mathrm{Ca}^{2+}$ wave, as we find that the $\operatorname{Ins}(1,4,5) P_{3}$ receptor ITR-1 is required for epidermal $\mathrm{Ca}^{2+}$ responses. We also identify an epidermal signal transduction pathway that includes the epidermal TRPM channel GTL-2, the G $\alpha_{\mathrm{q}}$ EGL-30 and its effector PLC $\beta$ EGL-8 as required for epidermal $\mathrm{Ca}^{2+}$ signaling. Epidermal activity of GTL-2 has also recently been implicated in regulating neuronal excitability, ${ }^{16}$ raising the possibility that woundtriggered epidermal $\mathrm{Ca}^{2+}$ elevation could also have non-autonomous effects.

A signaling cascade involving GPA-12 had been implicated in the epidermal innate immune response to damage. ${ }^{17}$ Wounding may trigger parallel $\mathrm{G}$ protein pathways involving GPA-12 and EGL-30. Extrapolating from these studies, one or more $\mathrm{G}$ protein coupled receptors (GPCR) may be involved in the initial activation of $\mathrm{G}$ protein signals by damage. Although the $C$. elegans genome encodes almost 1,500 putative GPCRs, most are nematodespecific chemoreceptors. ${ }^{18}$ Putative damage or tension-sensing GPCRs might be found by screening the smaller subset of nonchemoreceptor families. Alternatively, epidermal $G$ protein cascades might be activated in receptor-independent mechanisms reminiscent of those discovered in the early C. elegans embryo. ${ }^{19}$

\section{Calcium Signals Trigger Actin-Dependent Wound Closure}

The actin cytoskeleton is critical for wound closure in many models. ${ }^{20}$ Our study suggests $\mathrm{Ca}^{2+}$ signals are required for wound closure in the adult $C$. elegans epidermis, likely promoting direct actin polymerization (Fig. 2B). Small GTPases of the Rho family regulate the formation of actin structures such as actin cable, filopodia and lamellipodia. ${ }^{21}$ Drosophila embryonic wounds are closed by a contractile actomyosin cable, which acts like a purse string, and whose contractility is dependent on the Rho GTPase, Rho $1 .^{20}$ Unexpectedly, although contractile actin cables appear to form after wounding C. elegans skin, our results suggest that a "purse-string" closure mechanism is not essential for wound closure. In animals with reduced activity of Rho or of nonmuscle myosin II, the number of filopodia is increased but the area of actin accumulation is reduced (Fig. 2C), suggesting that protrusive activity is increased and that the wound can close faster than in the wild type. Purse-string dependent closure may be more critical in wounds of multicellular epithelia than in the syncytial epidermal wounds in C. elegans. ${ }^{22}$ Nevertheless our results suggest that changes in filopodia number and shape may be an alternative, compensatory mechanism for skin wounds to close, in the absence of actin cables.

In either case, actin polymerization is an essential initial step for wound closure. The small GTPase CDC-42 is essential for actin polymerization after wounding (Fig. 2C). Loss of the actin nucleating factors ARX-2 (ARP2/3) or WSP-1 (WASP), ${ }^{23}$ causes decreased formation of filopodia and slower wound closure, suggesting that in the absence of filopodial protrusions, actin cables are less efficient at closing the wound. Indeed, actin cables and filopodial protrusion appear to have opposing roles, in that wounds close faster by filopodial protrusion when the pursestring is removed. These observations raise the question of why opposing mechanisms might be activated by wounding; possibly a balance between filopodial protrusion and purse-string mediated closure is important for other aspects of wound healing such as scar formation. Wounding of single cells activates both Cdc42 and Rho, via a $\mathrm{Ca}^{2+}$ dependent signal. ${ }^{24}$ We speculate that wounding the C. elegans epidermis also triggers local activation of these small GTPases. If so, an important future goal will be to define factors responsible for GTPase activation in response to wounding, and to understand how these themselves are regulated in the epidermis.

\section{Remodeling, Scar Formation and Repair of the Permeability Barrier}

The later stages of wound repair, known as remodeling in mammalian models, involve reformation of the extracellular matrix and permeability barrier and the formation of scars. ${ }^{3,25}$ Intriguingly, scar-like autofluorescent structures are also seen after needle or laser wounding adult C. elegans epidermis (Fig. 2B), and are visible for several days at the wound site. ${ }^{9}$ The exact origin or composition of these structures remains to be determined; they form independently of the PMK-1 p38 cascade. Ultrastructural analysis reveals that scar structures are associated with thickened basal layers of cuticle, suggesting that wounding might induce local secretion of cuticle during repair. Interestingly, dapk-1 mutants display progressive hypertrophy of cuticle at the head region, including local accumulation of cuticle collagens and spontaneous autofluorescent scar-like material. ${ }^{13}$ As DAPK-1 can negatively regulate wound closure, DAPK-1 may provide a genetic entry point to understand how scar formation, cuticle secretion, and wound closure are coordinately regulated after wounding. Remarkably, mutations in sydn-1, a regulator of mRNA polyadenylation, ${ }^{26}$ strongly and specifically suppress these aspects of the Dapk-1 epidermal phenotype. The mechanism of SYDN-1 suppression may shed light on DAPK-1's functions in the epidermis, as may the identity of other 


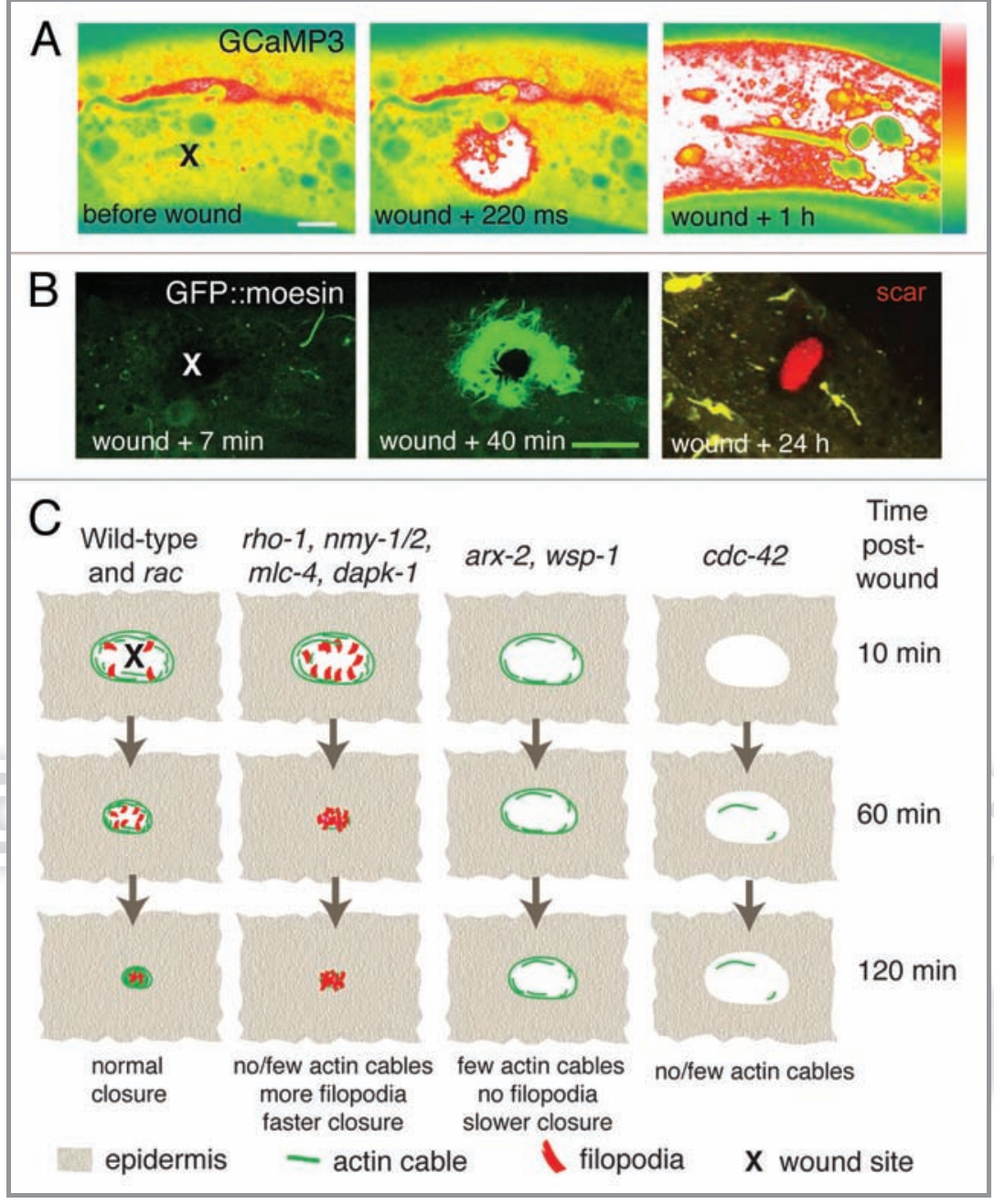

Figure 2. C. elegans epidermal wound responses. (A) Epidermal GCaMP fluorescence elevation after femtosecond laser wounding. Pcol-19-GCaMP3(juls319). Lateral views of adult epidermis in mid-body before, immediately after and $1 \mathrm{~h}$ after laser wounding; $\mathrm{x}$ marks site of laser wound. Spinning disk confocal, intensity code; scale, $10 \mu \mathrm{m}$. (B) Needle wounding triggers actin assembly around the wound site. Pcol-19-GFP::Moesin (juls352) labels actin filaments in adult epidermis. At $24 \mathrm{~h}$ an autofluorescent "scar" (red) is visible at the wound site and the actin structures have disappeared. $\mathrm{x}$ marks site of needle wound; laser scanning confocal images; scale, $10 \mu \mathrm{m}$. C, Graphical summary of mutant or RNAi phenotypes of genes implicated in C. elegans wound-induced actin dynamics.

as-yet uncloned suppressors of dapk-1 (T.I.H. and A.D.C., unpublished).

\section{Wounding and Gene Expression}

In C. elegans, needle or laser wounding triggers the elevated transcription of a large number of genes encoding AMPs. The Stat-like transcription factor STA-2 is

\section{References}

1. $\mathrm{Xu} \mathrm{S}$, Chisholm ADA. A G $\alpha \mathrm{q}-\mathrm{Ca}^{2+}$ signaling pathway promotes actin-mediated epidermal wound closure in C. elegans. Curr Biol 2011; 21:1960-7; PMID:22100061; http://dx.doi.org/10.1016/j.cub.2011.10.050 required for AMP induction in response to wounding ${ }^{27}$ and is localized to endocytic vesicles in the epidermis. It will be interesting to determine whether localization of these potential signaling vesicles is affected by wounding. It will also be important to address whether transcriptional regulators implicated in wound responses in other animals also play

2. Sonnemann KJ, Bement WM. Wound repair: toward understanding and integration of single-cell and multicellular wound responses. Annu Rev Cell Dev Biol 2011; 27:237-63; PMID:21721944; http://dx.doi.org/ 10.1146/annurev-cellbio-092910-154251 roles in $C$. elegans wound responses. For example, in mammals, the JUN $\mathrm{N}$-terminal kinase (JNK) and the transcription factor activator protein 1 (AP1) regulate gene expression after wounding. ${ }^{28}$ The function of AP1 in wound healing is conserved in Drosophila and mammals. ${ }^{29}$ Another transcription factor, Grainy head, has a remarkably conserved role in epidermal wound repair..$^{30,31}$ The C. elegans Grainy head ortholog GRH-1 is expressed in the epidermis and is required for embryonic cuticle integrity. ${ }^{32}$ It seems likely that GRH-1 is involved in epidermal wound healing in adults, although this remains to be explored. Gene expression profiling of wounded animals may be feasible, allowing a comprehensive analysis of the epidermal transcriptome's response to damage.

\section{Outlook}

The C. elegans epidermis has a robust repair mechanism that is likely critical for survival in an environment rich in skin-penetrating pathogens and sources of mechanical damage. We end by noting that many interesting areas of wound healing biology remain to explored in C. elegans. For example, what is the role of the lipid-rich epicuticle in the permeability barrier; is it resynthesized after wounding? How does aging affect wound repair in C. elegans? Do C. elegans embryos repair skin wounds, and if so do the mechanisms resemble those of the adult? Finally, does epidermal damage trigger other organismal responses, for example in the nervous system? The relative simplicity of $C$. elegans and its famous genetic tractability suggest that rapid progress should be possible in understanding such aspects of wound repair.

\section{Acknowledgments}

Our studies of epidermal development and wound repair are supported by NIH R01 GM054657.

3. Gurtner GC, Werner S, Barrandon Y, Longaker MT Wound repair and regeneration. Nature 2008; 453:314-21; PMID:18480812; http://dx.doi.org/10. 1038/nature07039 
4. Razzell W, Wood W, Martin P. Swatting flies: modelling wound healing and inflammation in Drosophila. Dis Model Mech 2011; 4:569-74; PMID: 21810906; http://dx.doi.org/10.1242/dmm.006825

5. Elias PM, Friend DS. The permeability barrier in mammalian epidermis. J Cell Biol 1975; 65:180-91; PMID:1127009; http://dx.doi.org/10.1083/jcb.65.1. 180

6. Koster MI, Roop DR. Mechanisms regulating epithelial stratification. Annu Rev Cell Dev Biol 2007; 23 93-113; PMID:17489688; http://dx.doi.org/10.1146/ annurev.cellbio.23.090506.123357

7. Page AP, Johnstone IL. The cuticle. WormBook 2007; 1-15; PMID:18050497

8. Couillault C, Pujol N, Reboul J, Sabatier L, Guichou JF, Kohara Y, et al. TLR-independent control of innate immunity in Caenorhabditis elegans by the TIR domain adaptor protein TIR-1, an ortholog of human SARM. Nat Immunol 2004; 5:488-94; PMID:15048112; http://dx.doi.org/10.1038/ni1060

9. Pujol N, Cypowyj S, Ziegler K, Millet A, Astrain A, Goncharov A, et al. Distinct innate immune responses to infection and wounding in the $C$. elegans epidermis. Curr Biol 2008; 18:481-9; PMID:18394898; http:// dx.doi.org/10.1016/j.cub.2008.02.079

10. Zugasti O, Ewbank JJ. Neuroimmune regulation of antimicrobial peptide expression by a noncanonical TGF-beta signaling pathway in Caenorhabditis elegans epidermis. Nat Immunol 2009; 10:249-56; PMID: 19198592; http://dx.doi.org/10.1038/ni.1700

11. Martin P, Parkhurst SM. Parallels between tissue repair and embryo morphogenesis. Development 2004; 131:3021-34; PMID:15197160; http://dx.doi.org/10. $1242 / \mathrm{dev} .01253$

12. Roupé KM, Nybo M, Sjöbring U, Alberius P, Schmidtchen A, Sørensen OE. Injury is a major inducer of epidermal innate immune responses during wound healing. J Invest Dermatol 2010; 130:1167-77; PMID:19727116; http://dx.doi.org/10.1038/jid.2009. 284

13. Tong A, Lynn G, Ngo V, Wong D, Moseley SL, Ewbank JJ, et al. Negative regulation of Caenorhabditis elegans epidermal damage responses by death-associated protein kinase. Proc Natl Acad Sci U S A 2009; 106:1457-61; PMID:19164535; http://dx.doi.org/10. 1073/pnas.0809339106
14. Stanisstreet M. Calcium and wound healing in Xenopus early embryos. J Embryol Exp Morphol 1982; 67:195205; PMID:6806425

15. McNeil PL, Steinhardt RA. Plasma membrane disruption: repair, prevention, adaptation. Annu Rev Cell Dev Biol 2003; 19:697-731; PMID:14570587; http://dx doi.org/10.1146/annurev.cellbio.19.111301.140101

16. Stawicki TM, Zhou K, Yochem J, Chen L, Jin Y. TRPM channels modulate epileptic-like convulsions via systemic ion homeostasis. Curr Biol 2011; 21:883 8; PMID:21549603; http://dx.doi.org/10.1016/j.cub. 2011.03.070

17. Ziegler K, Kurz CL, Cypowyj S, Couillault C, Pophillat $\mathrm{M}$, Pujol N, et al. Antifungal innate immunity in C. elegans: PKC $\delta$ links G protein signaling and conserved p38 MAPK cascade. Cell Host Microbe 2009; 5:341-52; PMID:19380113; http://dx.doi.org/ 10.1016/j.chom.2009.03.006

18. Thomas JH, Robertson HM. The Caenorhabditis chemoreceptor gene families. BMC Biol 2008; 6:42; PMID:18837995; http://dx.doi.org/10.1186/1741 7007-6-42

19. Hampoelz B, Knoblich JA. Heterotrimeric G proteins new tricks for an old dog. Cell 2004; 119:453-6; PMID:15537535; http://dx.doi.org/10.1016/j.cell. 2004.10.025

20. Wood W, Jacinto A, Grose R, Woolner S, Gale J Wilson $\mathrm{C}$, et al. Wound healing recapitulates morphogenesis in Drosophila embryos. Nat Cell Biol 2002; 4:907-12; PMID:12402048; http://dx.doi.org/10. $1038 / \mathrm{ncb} 875$

21. Jaffe $A B$, Hall A. Rho GTPases: biochemistry and biology. Annu Rev Cell Dev Biol 2005; 21:247-69; PMID:16212495; http://dx.doi.org/10.1146/annurev. cellbio.21.020604.150721

22. Kiehart DP. Wound healing: The power of the purse string. Curr Biol 1999; 9:R602-5; PMID:10469588; http://dx.doi.org/10.1016/S0960-9822(99)80384-4

23. Chhabra ES, Higgs HN. The many faces of actin: matching assembly factors with cellular structures. Nat Cell Biol 2007; 9:1110-21; PMID:17909522; http:// dx.doi.org/10.1038/ncb1007-1110
24. Benink HA, Bement WM. Concentric zones of active RhoA and Cdc42 around single cell wounds. J Cell Biol 2005; 168:429-39; PMID:15684032; http://dx.doi. org/10.1083/jcb.200411109

25. Clark AG, Miller AL, Vaughan E, Yu HY, Penkert R, Bement WM. Integration of single and multicellular wound responses. Curr Biol 2009; 19:1389-95; PMID 19631537; http://dx.doi.org/10.1016/j.cub.2009.06.044

26. Van Epps H, Dai Y, Qi Y, Goncharov A, Jin Y. Nuclear pre-mRNA 3'-end processing regulates synapse and axon development in C. elegans. Development 2010; 137:2237-50; PMID:20530551; http://dx.doi org/10.1242/dev.049692

27. Dierking K, Polanowska J, Omi S, Engelmann I, Gut $\mathrm{M}$, Lembo F, et al. Unusual regulation of a STAT protein by an SLC6 family transporter in C. elegan epidermal innate immunity. Cell Host Microbe 2011 9:425-35; PMID:21575913; http://dx.doi.org/10.1016/ j.chom.2011.04.011

28. Martin P, Nobes CD. An early molecular component of the wound healing response in rat embryos-induction of c-fos protein in cells at the epidermal wound margin. Mech Dev 1992; 38:209-15; PMID:1457382; http:// dx.doi.org/10.1016/0925-4773(92)90054-N

29. Schäfer M, Werner S. Transcriptional control of wound repair. Annu Rev Cell Dev Biol 2007; 23:69-92; PMID: 17474876; http://dx.doi.org/10.1146/annurev.cellbio. 23.090506.123609

30. Mace KA, Pearson JC, McGinnis W. An epidermal barrier wound repair pathway in Drosophila is mediated by grainy head. Science 2005; 308:381-5; PMID: 15831751; http://dx.doi.org/10.1126/science.1107573

31. Ting SB, Caddy J, Hislop N, Wilanowski T, Auden A, Zhao LL, et al. A homolog of Drosophila grainy head is essential for epidermal integrity in mice. Science 2005; 308:411-3; PMID:15831758; http://dx.doi.org/10. 1126/science. 1107511

32. Venkatesan K, McManus HR, Mello CC, Smith TF, Hansen U. Functional conservation between members of an ancient duplicated transcription factor family, LSF/ Grainyhead. Nucleic Acids Res 2003; 31:4304-16; PMID:12888489; http://dx.doi.org/10.1093/nar/gkg644 